\title{
Mazur and Golomb Awarded National Medal of Science
}

On December 21, 2012, President Obama announced the recipients of the National Medal of Science for 2011. Among the twelve honorees are two mathematicians: BARRY MAZUR of Harvard University and SOLOMON GOLOMB of the University of Southern California.

\section{Barry Mazur}

Barry Mazur was honored for "original and landmark contributions to differential topology, number theory, and arithmetic algebraic geometry, where, among other applications, his work was foundational to Wiles' proof of Fermat's last theorem and for his dedication to communicating subtle mathematical ideas to the broader public."

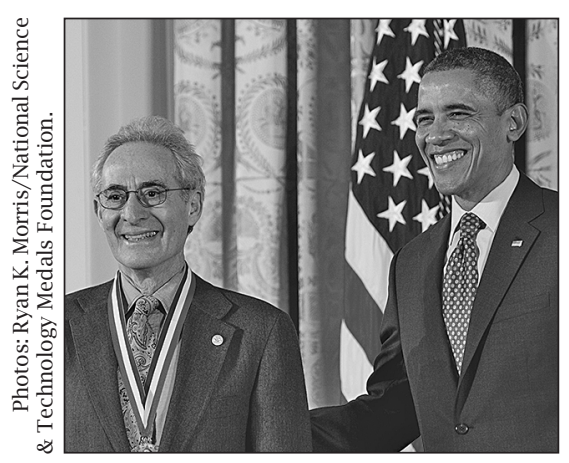

The Notices asked D. Kotschick of LudwigMaximilians-Universität München and R. L. Taylor of the Institute for Advanced Study to comment on the work of Mazur. They responded: "Barry Mazur is a mathematician of extraordinary breadth and originality. He started his career in topology, where he discovered what are now called Mazur manceremony, December 2012. include a proof of the generalized Schoenflies theorem and a proof of the $s$-cobordism theorem. Mazur was the first to formalize certain arguments using infinite repetitions, which are designed to push difficulties off to infinity. This is now referred to as the 'Mazur swindle'.

"In the late 1960s Mazur's interests evolved toward number theory. Here he made a series of fundamental contributions, such as his work on Katz's conjecture, his classification of the possible rational torsion points on an elliptic curve, and his proof (with Wiles) of the main conjecture of Iwasawa theory. Mazur has repeatedly introduced

DOI: http://dx.doi.org/10.1090/noti1020 new ideas and techniques into number theory that have changed the direction of the subject. With Swinnerton-Dyer he was the first to introduce $p$-adic $L$-functions for modular forms, a subject that has received enormous attention ever since. His 1977 Eisenstein ideal paper marked a turning point in the number theoretic applications of the detailed arithmetic algebraic geometry of modular curves. In the mid-1980s Mazur introduced the deformation theory of Galois representations, a simple, but extremely powerful, idea that transformed number theory. This proved to be key to Wiles' proof of Fermat's last theorem. A few years later, with Coleman, he introduced many new $p$-adic families of modular forms ('the eigencurve'), which continue to be a major topic of research. As important as Mazur's mathematical contributions are his remarkable contributions as a mentor. He has advised dozens of graduate students and postdocs, including many of the most successful number theorists of the last thirty-five years."

Barry Mazur was born in New York City and, under the direction of Ralph Fox, received his Ph.D. from Princeton University in 1959. At the time he received his degree, he had already proven the generalized Schoenflies conjecture in geometric topology. He was a research fellow at the Institute for Advanced Study from 1958 to 1959, and in 1959 he published his first four papers. He was a Junior Fellow at Harvard from 1959 to 1962. He became an assistant professor at Harvard in 1962 and is currently the Gerhard Gade University Professor and a Senior Fellow. His other honors include the Veblen Prize (1965), the Cole Prize (1982), the Chauvenet Prize (1994), and the Steele Prize (1999). He was elected a member of the National Academy of Sciences in 1982 and of the American Philosophical Society in 2001. He is the author of the book Imagining Numbers (Particularly the Square Root of Minus Fifteen) (Farrar, Straus and Giroux, 2003), and coauthor of Universal Extensions and One Dimensional Crystalline Cohomology with William Messing (Lecture Notes in Mathematics, Springer, 1974) and of Kolyvagin Systems with Karl Rubin (Memoirs of the AMS, 2004). 


\section{Solomon W. Golomb}

Golomb was honored with the National Medal of Science for his "pioneering work in shift register sequences that changed the course of communications from analog to digital and for numerous innovations in reliable and secure space, radar, cellular, wireless and spread-spectrum communications."

The Notices asked Alfred W. Hales to comment on the work of Golomb. Hales is a professor emeritus at the University of California Los Angeles and is currently with the Center for Communications Research of the Institute for Defense Analyses. Hales responded: "For the past fifty-plus years, Solomon Golomb has been a world leader in the development and application of mathematics for communications and coding theory, especially digital and space communications. In his remarkable career, first at the Jet Propulsion Laboratory and then at the University of Southern California (in electrical engineering and mathematics), his research contributions have ranged over a wide spectrum of science and technology. Perhaps he is best known for his mathematical analysis of shift register sequences in his classic book with that title and in numerous journal publications-such sequences are ubiquitous in radar, space communications, cryptography, cell phones, etc. Other noteworthy contributions in this direction include Golomb (entropy) coding, Golomb rulers, and the Golomb construction for Costas arrays. For all this work the IEEE has honored him with both its Hamming Medal and the Shannon Award in Information Theory. Golomb's Harvard Ph.D. was in analytic number theory, and he has extensive (and seminal) publications in number theory, combinatorics, algebra, and various other fields (including even molecular genetics). He is also a noted expert in mathematical game theory (polyominoes were his invention), and he continues to publish a number of puzzle columns in various journals. In addition to his many research contributions, Golomb has had a great influence on future generations through his generous and insightful mentoring of young people-his students, postdocs, and many others."

Solomon Golomb was born in Baltimore, Maryland. He received his B.A. from Johns Hopkins University and his Ph.D. in mathematics from Harvard University in 1957 under the direction of David Widder. He is currently professor of electrical engineering at the University of Southern California and is best known to the general public and fans of mathematical games as the inventor of polyominoes, the inspiration for the computer game Tetris. While he was working toward his Ph.D. he held a Fulbright Fellowship at the University of Oslo. He worked at the Jet Propulsion Laboratory at the California Institute of Technology from 1956 to 1963 , where he was a senior research mathematician and later supervisor of the research group and assistant chief of the Telecommunications Research Section. In the latter position he played a key role in formulating the design of deep-space communications for the subsequent lunar and planetary explorations. He joined the University of Southern California in 1963.

In 1992 he received the medal of the U.S. National Security Agency for his re-

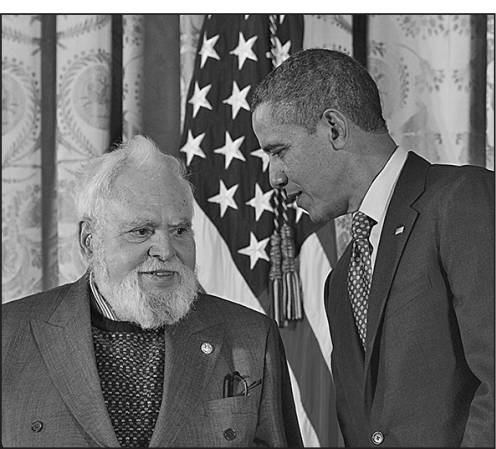

Solomon Golomb and President Obama. search. He has also been the recipient of the Lomonosov Medal of the Russian Academy of Science and the Kapitsa Medal of the Russian Academy of Natural Sciences. His honors from the IEEE include the Shannon Award (1985) and the Richard W. Hamming Medal (2000) for his exceptional contributions to information sciences and systems. He was awarded the USC Presidential Medallion in 1985. He has been a major contributor in coding and information theory for more than forty years and is recognized for his ability to apply advanced mathematics to problems in digital communications. His books include Shift Register Sequences (Holden-Day, 1967), Polyominoes (Princeton University Press, 2nd ed., 1996), and Signal Design for Good Correlation (coauthored with Guang Gong, Cambridge University Press, 2005). He is a member of the National Academy of Engineering, the Institute of Electrical and Electronics Engineers (IEEE), the American Association for the Advancement of Science (AAAS), and a fellow of the AMS. He became a foreign member of the Russian Academy of Natural Science in 1994.

\section{About the Medal}

The National Medal of Science is the country's highest distinction for contributions to scientific research. According to a news release from the Office of Science and Technology Policy, "The National Medal of Science honors individuals for pioneering scientific research in a range of fields, including physical, biological, mathematical, social, behavioral, and engineering sciences, that enhances our understanding of the world and leads to innovations and technologies that give the United States its global economic edge." The National Science Foundation administers the award, which was established by Congress in 1959.

- Elaine Kehoe 